\title{
USP28 promotes aerobic glycolysis of colorectal cancer by increasing stability of FOXC1
}

\author{
Zhaohui Liu', Min Chen ${ }^{2 \Xi}$, Xiaoping Xu' ${ }^{1}$, Lei Zhang ${ }^{1}$, Yuan Pan ${ }^{1}$ and Dong Chen ${ }^{1}$ \\ 'Department of Anorectal Surgery, First People's Hospital of Yuhang District, Hangzhou, Hangzhou City, Zhejiang Province, 311100, P.R. China; \\ 2Department of Anesthesia, First People's Hospital of Yuhang District, Hangzhou, Hangzhou City, Zhejiang Province, 311100 , P.R. China
}

\begin{abstract}
Aerobic glycolysis is essential for cancer cell metabolism and growth. Deubiquitinase, USP28 (ubiquitin specific peptidase 28), could maintain stability of proteins involved in tumor progression. This study was performed to investigate the role of USP28 in aerobic glycolysis of colorectal cancer. Our data showed that USP28 mRNA and protein expressions were enhanced in colorectal cancer tissues and cells. Functional assays demonstrated that overexpression of USP28 promoted cell proliferation and aerobic glycolysis of colorectal cancer, while USP28 inhibition could reverse these effects. Protein expression of Forkhead Box C1 (FOXC1) was increased by USP28 over-expression, whereas knockdown of USP28 aggravated cycloheximide ( $\mathrm{CHX}$; protein synthesis inhibitor) stimulated decrease of FOXC1. Moreover, proteasome inhibitor, MG132, could rescue USP28 silence-induced degradation of FOXC1. Overexpression of FOXC1 counteracted the suppressive effects of USP28 interference on colorectal cancer cell viability and aerobic glycolysis. In conclusion, USP28 enhanced cell viability and aerobic glycolysis of colorectal cancer by stabilizing FOXC1, suggesting that USP28-FOXC1 might be a novel therapeutic avenue for colorectal cancer.
\end{abstract}

Keywords: USP28, FOXC1, aerobic glycolysis, stability, colorectal cancer

Received: 25 September, 2020; revised: 09 December, 2020 accepted: 27 January, 2021; available on-line: 09 June, 2021

⿶e-mail: min_chen1234@163.com

Abbreviations: $\mathrm{CHX}$, cycloheximide; FBP1, fructose-1,6-bisphosphatase 1; FOXC1, Forkhead Box C1; qRT-PCR, quantitative reverse transcriptase PCR; USP28, ubiquitin specific peptidase 28

\section{INTRODUCTION}

Colorectal cancer is one of the most common cancers in the world (Yang, 2019). Although 5-year survival rate of colorectal cancer patients is improved via surgical resection, radiotherapy and systemic chemotherapy at an early stage, high recurrence rate caused by distant metastasis still threatens a large proportion of advanced stage patients (Prenen et al.,, 2013). Recently, efforts have been made to elucidate the genetic and molecular characteristics of colorectal cancer for better prognosis and response to targeted therapies (Fleshman \& Smallwood, 2015). Understanding the mechanisms involved in cell proliferation and metastasis of colorectal cancer might also improve treatment of the patients.

Tumor cells undergo abnormal glycolysis, accompanied by increased glucose uptake and lactic acid production, that is known as aerobic glycolysis or Warburg effect (Lu, 2019). Aerobic glycolysis plays an essential role in tumor cell proliferation with the synthesis of nucleic acids, amino acids and fats, and the production of lactic acid in microenvironment that could enhance tumor metastasis and treatment resistance (Doherty \& Cleveland, 2013). Activation of aerobic glycolysis has been shown to be associated with colorectal cancer metastasis and poor prognosis of the patients (Satoh et al., 2017). Therefore, inactivation of glycolysis might facilitate for suppression of colorectal cancer metastasis (Hu et al., 2020).

Ubiquitin specific peptidase 28 (USP28), member of USPs, contains a ubiquitin-recognition site and corresponding catalytic mechanism (Wang et al., 2018). With the ability to regulate physiological homeostasis of ubiquitination process, tune DNA-damage response and maintain cell cycle, targeting USP28 has shown clinical significance in cancer therapy (Wang et al., 2018). For example, USP28 could increase the characteristics of breast cancer stem cells by deubiquitinating lysine-specific demethylase 1 (Wu et al., 2013). Inhibition of USP28 promotes degradation of lysine-specific demethylase 1 to repress gastric cancer cell metastasis (Chen et al., 2018). In colorectal cancer, USP28 could regulate intestinal homeostasis and promote the progression of intestinal diseases and tumor progression (Diefenbacher et al., 2014; Diefenbacher et al., 2015). Inhibitor of USP28 could reduce cancer-related proteins, followed by suppressing colorectal cancer progression (Wang et al., 2020). However, the mechanism of USP28 in colorectal cancer aerobic glycolysis has not been reported yet.

This study was performed to explore the role and molecular mechanism of USP28 in aerobic glycolysis of colorectal cancer. Results showed that USP28 could promote aerobic glycolysis of colorectal cancer and promote the cell growth through enhanced stability of Forkhead Box C1 (FOXC1), thus providing a novel therapeutic avenue for colorectal cancer.

\section{MATERIALS AND METHODS}

\section{Tissue specimens and immunohistochemistry}

Sixty-three colorectal patients were recruited from First People's Hospital of Yuhang District, Hangzhou. The tumor tissues and adjacent healthy tissues were acquired from patients with informed consent. Ethics approval was acquired from the Ethics Committee of First People's Hospital of Yuhang District, Hangzhou. For immunohistochemical analysis, paraffin-embedded colorectal tumor tissues were cut into $4 \mu \mathrm{m}$ sections. Sections were deparaffinized, dehydrated, blocked, and incubated with primary anti-USP28 antibody (1:100; Miltenyi Biotech, Belgium, Germany). The sections were 
incubated with horseradish peroxidase-labeled secondary antibody (Abcam, Cambridge, UK) followed by staining with diaminobenzidine and counterstaining with hematoxylin. Slides were visualized under a light microscope (Olympus, Tokyo, Japan).

\section{Cell culture, treatment and transfection}

Different cell lines T84, LoVo, HCT116, HCT8, SW480 and NCM460 (ATCC, Manassas, VI, USA) were cultured in DMEM (Invitrogen, Carlsbad, CA, USA) containing $10 \%$ fetal bovine serum (Invitrogen) at $37^{\circ} \mathrm{C}$. Full length of USP28 and FOXC1 were inserted into pcDNA3.1 (Invitrogen). USP28 shRNAs (shUSP28\#1 or 2\#) were acquired from GenePharma (Suzhou, China). For cell transfection, T84 or SW480 were transfected with shRNAs or vectors using Lipofectamine 2000 (Invitrogen). For cell treatment, two days after T84 or SW480 transfections, cells were incubated with $50 \mu \mathrm{g} /$ $\mathrm{mL}$ CHX (Sigma Aldrich, St. Louis, MO, USA) for 0, 4, 8, 12 hours or $25 \mu \mathrm{M}$ MG132 (Sigma Aldrich) for 12 hours before western blot analysis.

\section{MTT and colony formation assays}

T84 or SW480 transfections were seeded into plates at the density of $4 \times 10^{3}$ cells/well at indicated time $(0$, 24, 48, 72, 96, 120 hours). MTT reagent (20 $\mu \mathrm{L}$; Sigma Aldrich) was added to the plates two hours before the measurement of absorbance at $490 \mathrm{~nm}$ by microplate spectrophotometer (Thermo Scientific, Waltham, MA, USA). For colony formation assay, T84 or SW480 transfections were seeded into plates at the density of $1 \times 10^{2}$ cells/well, and then cultured for 12 days. Colonies in each well were measured under microscope (Olympus) following fixation in methanol and staining in $0.05 \%$ crystal violet.

\section{Measurement of glucose uptake, lactate and ATP levels}

For glucose uptake analysis, T84 or SW480 transfections were seeded into plates for 48 hours. The cultured medium was collected and the cells were harvested. Protein concentration in cells was determined by Pierce bicinchoninic acid protein assay kit (Thermo Scientific) and the glucose concentration was determined by glucose assay kit (Shanghai Rongsheng Biotech, Shanghai, China). The values of glucose uptake were normalized to the total protein number. For lactate and ATP levels determination, the cultured medium was conducted with lactate or ATP assay kits (Nanjing KeyGen Biotech, Nanjing, China). The values of lactate and ATP levels were also normalized to the total protein number.

\section{Extracellular acidification rate (ECAR)}

XF Glycolytic Rate Assay kit (Agilent Technologies, Santa Clara, USA) with Seahorse Bioscience XF Extracellular Flux Analyzer (Seahorse Bioscience, North Billerica, USA) was used to determine ECAR for the evaluation of glycolytic function. T84 or SW480 was seeded into XF24 plates for 24 hours. XF Base Media $(5 \mathrm{mM}$ HEPES, $10 \mathrm{mM}$ glucose, $1 \mathrm{mM}$ sodium pyruvate, and $2 \mathrm{mM}$ L-glutamine) without phenol red was then used to detect the glycolytic rate under WAVE version 2.6.0 (Agilent Technologies). Oligomycin $(1 \mu \mathrm{M})$ and 2-deoxyglycose $(50 \mathrm{mM})$ were added as inhibitors into the plate.

\section{Western blot}

Total of $25 \mu \mathrm{g}$ proteins were separated by SDS-PAGE, and then transferred to polyvinylidene fluoride membrane (Bio-Rad, Hercules, CA, USA). Following block with 5\% skim milk, membrane was probed with antibodies, including anti-USP28 (1:2000, Abcam, Cambridge, UK), antiFOXC1 (1:2500, Abcam), anti-FBP1 (1:3000, Abcam), anti- $\beta$-actin (1:3500, Abcam) and anti-GAPDH (1:3500, Abcam). $\beta$-actin and GAPDH were used as the reference genes. Membrane was subsequently probed with horseradish peroxidase-labeled second antibody, and the bands were visualized using ECL Western blotting detection kits (Millipore, Billerica, MA, USA). The densitometry of the bands was quantified by Image J k 1.45 software.

\section{Quantitative reverse transcriptase PCR (qRT-PCR)}

Total RNA (1 $\mu \mathrm{g})$, isolated from frozen tissues by TRIzol reagent (Thermo Scientific), was reverse transcribed into cDNA under conditions: $37^{\circ} \mathrm{C} 15$ minutes, $85^{\circ} \mathrm{C} 5$ seconds and $4^{\circ} \mathrm{C} 10$ minutes. The cDNA was then conducted with qRT-PCR analysis with SYBR Premix Ex Taq kit (Takara, Otsu, Japan) under conditions: $95^{\circ} \mathrm{C} 5 \mathrm{~min}-$ utes; 45 cycles of $95^{\circ} \mathrm{C}$ for 10 seconds, $60^{\circ} \mathrm{C} 10$ seconds and $72^{\circ} \mathrm{C}$ for 10 seconds. The primer sequences were listed as below: USP28 (F: 5'-ATCTTCAGGCTGCCATTGCT-3' and R: 5'-CTAGCTGGAATGCGTCCTCT-3') and $\beta$-actin (F: 5'-TGCCCATCTACGAGGGGTATG-3' and R: 5'-CTCCT'TAATGTCACGCACGAT'TC-3').

\section{Statistical analysis}

Data were presented as mean \pm SEM from at least three independent experiments. Statistical analysis was conducted with SPSS 22.0, and evaluated by Student's ttests or one-way analysis of variance. $p<0.05$ was considered as statistically significant difference.

\section{RESULTS}

\section{Enhanced USP28 in colorectal cancer}

To evaluate the expression level of USP28 in colorectal cancer tissue, qRT-PCR and western blot analysis were performed. Results showed that mRNA (Fig. 1A) and protein (Fig. 1B and 1C) expression of USP28 were enhanced in the tumor tissues. Similarly, up-regulation of USP28 was also validated in colorectal cancer cells compared to NCM460 (normal human colon mucosal epithelial cell line) (Fig. 1D), suggesting potential regulatory role of USP28 on colorectal cancer.

\section{USP28 promoted colorectal cancer cell growth}

T84 was transfected with pcDNA-USP28 (Fig. 2A) and SW480 was transfected with shUSP28\#1 or 2\# (Fig. 2B) to exploit the functional role of USP28 on colorectal cancer. shUSP28\#1 showed lower expression of USP28 than shUSP28\#2 and was used for the following functional assays. Data from MTT and colony formation assays indicated that overexpression of USP28 promoted cell viability (Fig. 2C) and proliferation (Fig. 2D) of T84, while knockdown of USP28 reversed the promotive effects on cell viability (Fig. 2C) and proliferation (Fig. 2D) in SW480. These results indicated that USP28 promoted colorectal cancer cell growth. 
A

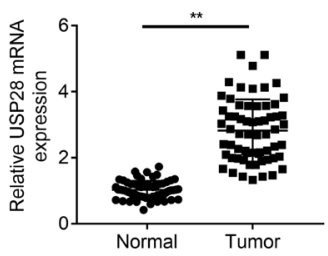

C
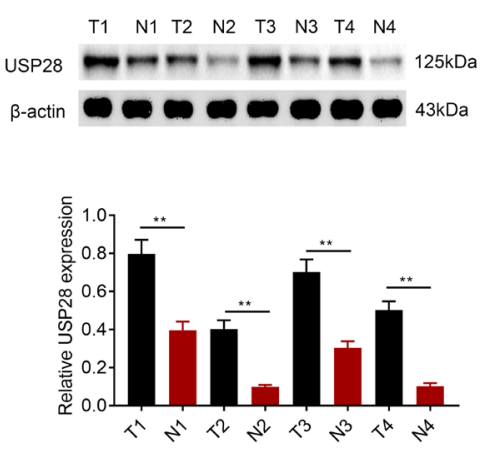

B

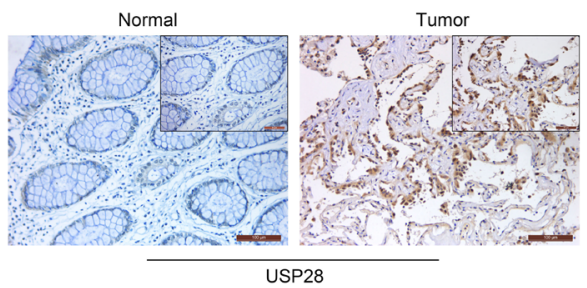

D
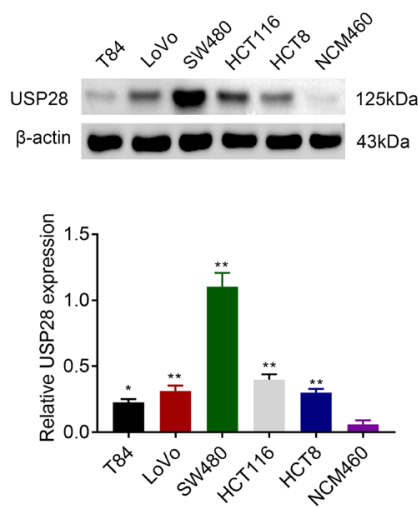

Figure 1. Enhanced USP28 in colorectal cancer

Increased mRNA expression of USP28 in colorectal cancer tissues compared to normal adjacent tissues. Immunohistochemical analysis of colorectal cancer tissues compared to normal adjacent tissues indicated the upregulation of USP28 in tumor tissues. Induced protein expression of USP28 in colorectal cancer tissues compared to normal adjacent tissues. Protein expression of USP28 was enhanced in colorectal cancer cells compared to NCM460 (normal human colon mucosal epithelial cell line). ${ }^{*} p<0.05 ;{ }^{* *} p<0.01$.

A
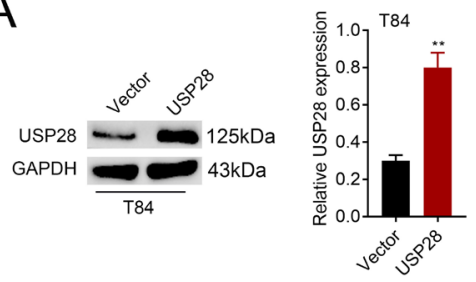

B

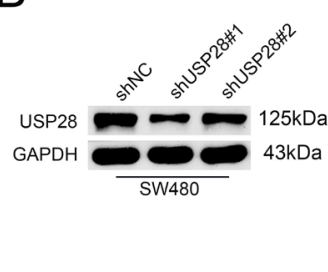

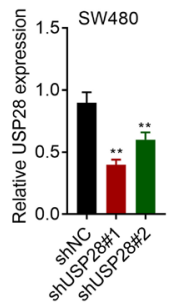

C
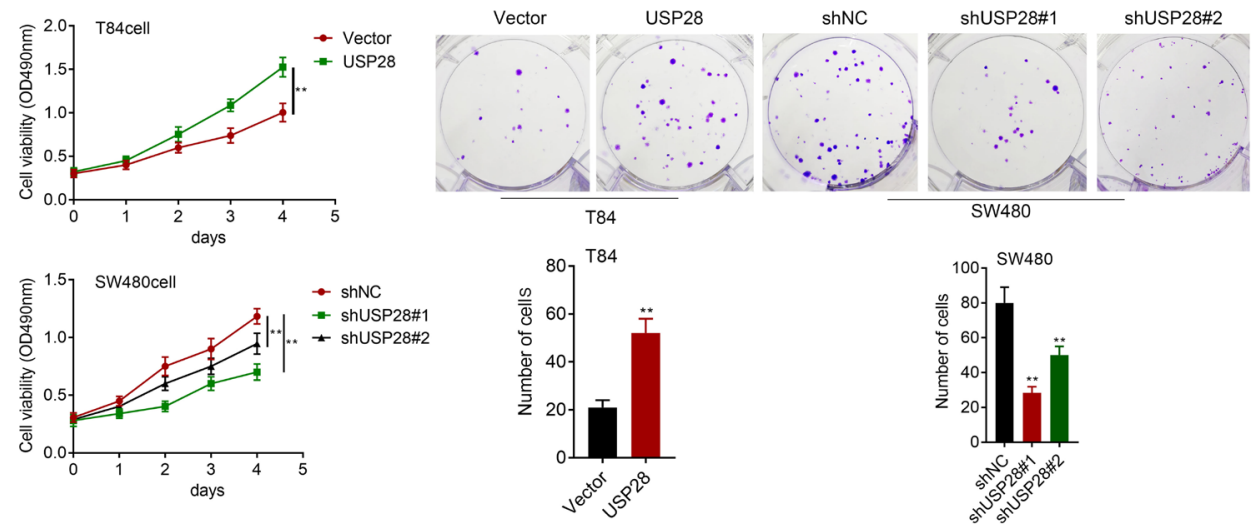

Figure 2. USP28 promoted colorectal cancer cell growth

Up-regulation of USP28 in T84 transfected with pCDNA-USP28. Down-regulation of USP28 in SW480 transfected with shUSP28 \#1 or 2\#. Increased cell viability in T84 was transfected with pcDNA-USP28 and decreased cell viability in SW480 transfected with shUSP28\# 1 and \#2. Increased cell proliferation in T84 was transfected with pcDNA-USP28 and decreased cell proliferation in SW480 transfected with shUSP28\#1 and \#2. ${ }^{* *} p<0.01$ 
A

B
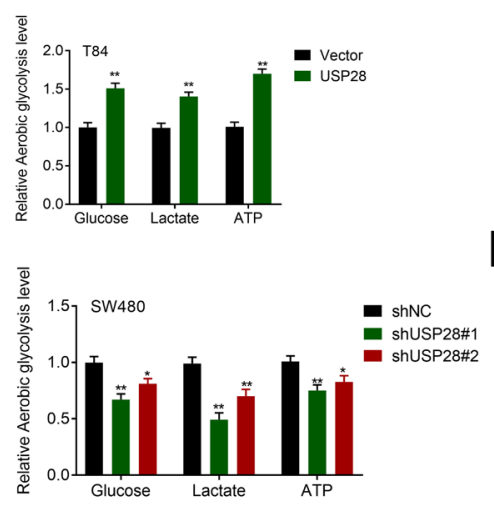

C

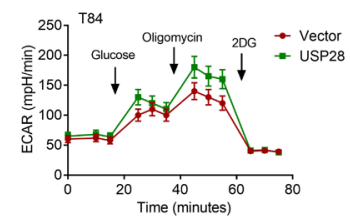

D

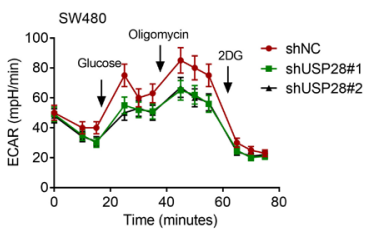

Figure 3. USP28 promoted aerobic glycolysis of colorectal cancer cell

Increased glucose uptake, lactate and ATP levels in T84 transfected with pcDNA-USP28. Decreased glucose uptake, lactate and ATP levels in SW480 transfected with shUSP28\#1 and \#2. Increased ECAR in T84 transfected with pcDNA-USP28. Decreased ECAR in SW480 transfected with shUSP28\#1 and \#2. ${ }^{* *} p<0.01$.

\section{USP28 promoted aerobic glycolysis of colorectal cancer cells}

The role of USP28 on colorectal cancer cell metabolism was then evaluated to examine the involvement of USP28 in colorectal tumor development. Energy production was induced by USP28 overexpression with increased glucose uptake, lactate and ATP levels in T84 (Fig. 3A). However, knockdown of USP28 decreased glucose uptake, lactate and ATP levels in SW480 (Fig. 3B). Moreover, USP28 overexpression enhanced the ECAR (Fig. 3C), while knockdown of USP28 reduced the ECAR (Fig. 3D), indicating the promotive effect of USP28 on aerobic glycolysis of colorectal cancer cell.

\section{USP28 promoted stability of FOXC1}

To uncover mechanism involved in USP28-mediated colorectal cancer progression, protein expression of USP28 was determined in T84 and SW480. Results revealed that overexpression USP28 increased FOXC1 expression in T84 (Fig. 4A), while USP28 knockdown decreased the expression (Fig. 4B). Expression of FOXC1 was time-dependently decreased in SW480 transfected with shNC or shUSP28\#1 post cycloheximide treatment (Fig. 4C). However, SW480 transfected with shUSP28\#1 demonstrated lower expression of USP28 compared to that with shNC (Fig. 4C). Moreover, proteasome inhibitor MG132 attenuated USP28 interference-induced decrease of FOXC1 (Fig. 4D), suggesting that USP28 might pro-

B
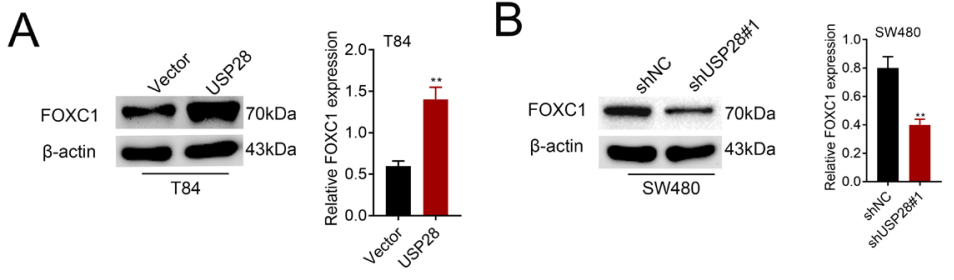

C
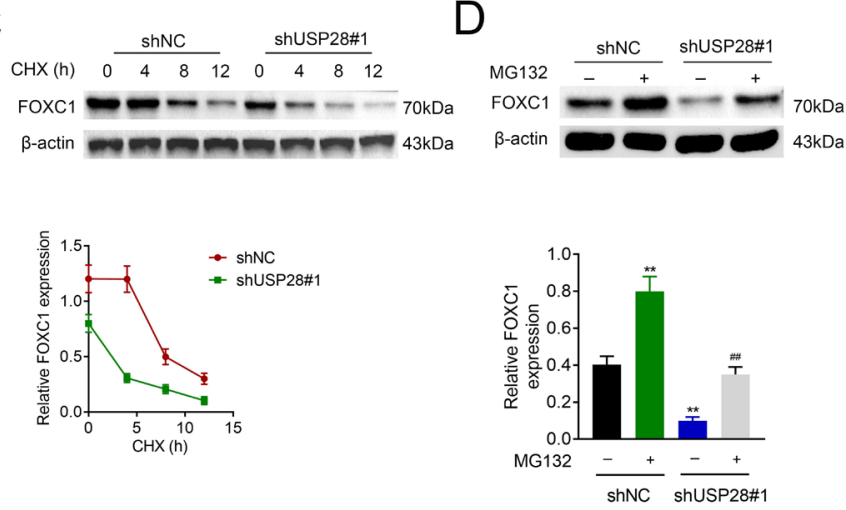

Figure 4. USP28 promoted stability of FOXC1

Increased FOXC1 expression in T84 was transfected with pCDNA-USP28. Decreased FOXC1 expression in SW480 transfected with shUSP28\#1. Decreased FOXC1 expression in SW480 transfected with shNC or shUSP28\#1 post cycloheximide treatment. Increased FOXC1 expression in SW480 transfected with shNC or shUSP28\#1 post MG132 treatment. ${ }^{* *}, \# \# p<0.01$. 
A

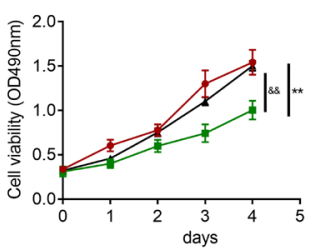

- shNC+Control - shUSP28\#1+Control - ShUSP28\#1+Control
- shUSP28\#1+FOXC1
B
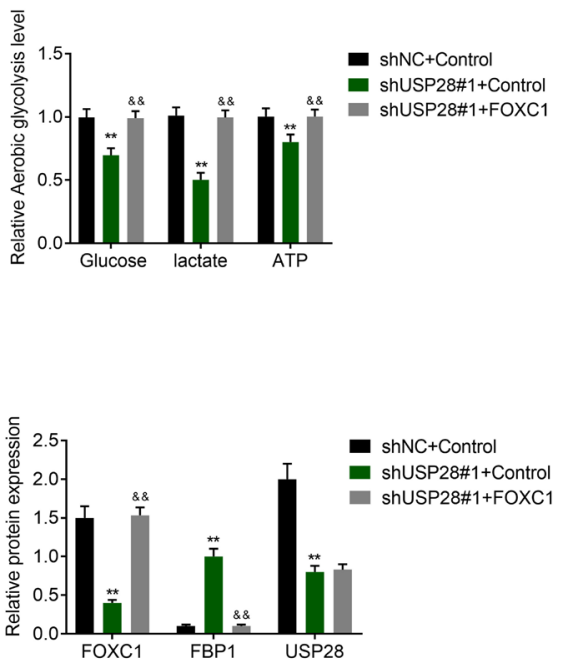

Figure 5. USP28 promoted growth and aerobic glycolysis of colorectal cancer cell through FOXC1

Decreased cell viability of SW480 induced by shUSP28\#1 was reversed by over-expression of FOXC1. Overexpression FOXC1 attenuated shUSP28\#1-induced decrease of glucose uptake, lactate and ATP levels. Knockdown of USP28 decreased FOXC1 to increase FBP1, and over-expression of FOXC1 counteracted with the promotive effect of USP28 silence on protein expression of FBP1. ${ }^{* *} \& \& p<0.01$.

mote stability of FOXC1 through inhibition of proteasome-mediated degradation in colorectal cancer cells.

\section{USP28 promoted growth and aerobic glycolysis of colorectal cancer cell through FOXC1}

To determine role of USP28/FOXC1 axis on colorectal cancer, SW480 was co-transfected with shUSP28\#1 or shUSP28\#2 and pcDNA-FOXC1. Decreased cell viability induced by knockdown of USP28 was reversed by overexpression of FOXC1 in SW480 (Fig. 5A and Supplementary Fig. S1A at https://ojs.ptbioch.edu.pl/ index.php/abp). FOXC1 Overexpression attenuated the decrease of glucose uptake, lactate and ATP levels by USP28 knockdown (Fig. 5B and Supplementary Fig. S1B at https://ojs.ptbioch.edu.pl/index.php/abp). Moreover, knockdown of USP28 could decrease FOXC1 to increase glycolytic enzyme, fructose-1,6-bisphosphatase 1 (FBP1), thus regulating the aerobic glycolysis (Fig. 5C). However, over-expression of FOXC1 counteracted the promotive effect of USP28 knockdown on protein expression of FBP1 (Fig. 5C). Therefore, USP28 could promote growth and aerobic glycolysis of colorectal cancer cell through up-regulation of FOXC1 and down-regulation of FBP1.

\section{DISCUSSION}

Cycles between ubiquitination and deubiquitination are essential for various biological processes, and the dysregulation of deubiquitination is involved in tumorigenesis (Sacco et al., 2010). For example, USP13 functioned as tumor-suppressor through stabilization of PTEN (Zhang et al., 2013). USP7 could stabilize Mdm2 to promote degradation of p53 (Sheng et al., 2006). Recently, deubiquitination by USP37 has been reported to regulate aerobic glycolysis of tumors (Pan et al., 2015). Considering the promotive effect of USP28 on colorectal (Diefenbacher et al., 2014; Diefenbacher et al., 2015), the role of USP28 on aerobic glycolysis of colorectal cancer was then evaluated in this study.
Previous study has shown that USP28 was enhanced in colorectal cancer tissues, and USP28 could form a positive feedback loop with oncoprotein, c-MYC, to promote colorectal cancer progression (Diefenbacher et al., 2014). Our results also showed that up-regulation of USP28 in colorectal cancer led to cell proliferation. Since cancer cells in aerobic glycolysis increased lactate production during the growth (Soga, 2013), the aerobic glycolysis in colorectal cancer was promoted by USP28 with increased glucose uptake, lactate and ATP production. As is well known, c-MYC is a transcriptional factor to regulate metabolic enzymes, such as hexokinase 2, phosphofructokinase and lactate dehydrogenase A, involved in aerobic glycolysis and tumor progression ( $\mathrm{He}$ et al., 2015). Inhibition of c-MYC could suppress aerobic glycolysis in colorectal cancer (Xu et al., 2015). USP28 could interact with ubiquitin ligase, F-box and WD repeat domain containing 7 and further stabilize c-MYC (Popov et al., 2007; Chakravorty et al., 2020). Silencing USP28 destabilized c-MYC in esophageal cancer cells (Weili et al., 2019). Therefore, the downstream target of USP28 involved in colorectal cancer progression needs to be further investigated.

FBP1, key glycolytic enzyme in aerobic glycolysis, was a target gene of FOXC1 to enhance aerobic glycolysis in colorectal cancer ( $\mathrm{Li}$ et al., 2019). Moreover, FOXC1 could bind to integrin $\alpha 7$ or fibroblast growth factor receptor 4 to promote colorectal cancer metastasis (Liu et al., 2018), and stabilization of FOXC1 could enhance the metastasis of colorectal cancer (Zhang et al., 2020). Therefore, we suggested that USP28 might promote aerobic glycolysis in colorectal cancer by stabilizing FOXC1. Knockdown of USP28 decreased expression of FOXC1 in colorectal cancer cells post protein synthesis inhibitor (cycloheximide) treatment. Moreover, proteasome inhibitor MG132 attenuated USP28 interference-induced decrease of FOXC1. These results suggested that USP28 regulated stability of FOXC1 depending on inhibition of proteasome-mediated degradation in colorectal cancer cells. Functional assays further indicated that over-expression of FOXC1 counteracted the suppressive 
effects of USP28 knockdown on cell viability and aerobic glycolysis of colorectal cancer. Moreover, in line with previous report that FOXC1 could down-regulate FBP1 in colorectal cancer cells ( $\mathrm{Li}$ et al., 2019), our results revealed that knockdown of USP28 decreased FOXC1 and increased FBP1, while over-expression of FOXC1 attenuated promotive effect of USP28 knockdown on protein expression of FBP1. Thus, USP28 could sustain growth and aerobic glycolysis of colorectal cancer cells through up-regulation of FOXC1 as well as down-regulation of FBP1. The present study for the first time showed that the deubiquitination of FOXC1 through USP28 could increase aerobic glycolysis in colorectal cancer. However, cycles of ubiquitination and deubiquitination have been shown to mediate activation of AKT (Yang et al., 2013), which regulated aerobic glycolysis in tumors (Elstrom et al., 2004), and phosphorylation of ser241 and ser272 of FOXC1 when p38 suppressed the ubiquitination and degradation of FOXC1 (Zhang et al., 2020). Therefore, whether AKT or p38 pathways were involved in USP28/FOXC1/FBP1 axis-mediated colorectal cancer aerobic glycolysis would be further investigated in further research.

\section{CONCLUSION}

In summary, the present study illustrated that USP28, overexpressed in colorectal cancer tissues, could promote cell proliferation and enhance the aerobic glycolysis of colorectal cancer cells. Mechanistically, USP28 could block the degradation of FOXC1 to down-regulate FBP1, thereby promoting aerobic glycolysis. Our data demonstrated that USP28 and FOXC1 might be the potential therapeutic targets in colorectal cancer due to the biological significance of USP28/FOXC1/FBP1 axis.

\section{Acknowledgements}

Not applicable.

\section{Funding}

Not applicable.

\section{Competing interests}

The authors state that there are no conflicts of interest to disclose.

\section{Ethics approval}

Ethical approval was obtained from the Ethics Committee of First People's Hospital of Yuhang District, Hangzhou.

\section{Statement of Informed Consent}

Written informed consent was obtained from a legally authorized representative(s) for anonymized patient information to be published in this article.

\section{Availability of data and materials}

All data generated or analyzed during this study are included in this published article.

\section{Authors' contributions}

Zhaohui Liu and Min Chen designed the study, supervised the data collection, Xiaoping $\mathrm{Xu}$ analyzed the data, interpreted the data, Lei Zhang, Yuan Pan and Dong Chen prepare the manuscript for publication and reviewed the draft of the manuscript. All authors have read and approved the manuscript.

\section{REFERENCE}

Chakravorty D, Ghosh A, Saha S (2020) Computational approach to target USP28 for regulating Myc. Comput Biol Chem 85: 107208. https://doi.org/10.1016/j.compbiolchem.2020.107208

Chen HS, Tong HS, Zhao Y, Hong CY, Bin JP, Su L (2018) Differential expression pattern of exosome long non-coding RNAs (lncRNAs) and microRNAs (miRNAs) in vascular endothelial cells under heat stroke. Med Sci Monit 24: 7965-7974. https://doi. org/10.12659/MSM.909983

Diefenbacher ME, Chakraborty A, Blake SM, Mitter R, Popov N, Eilers M, Behrens A (2015) Usp28 counteracts Fbw7 in intestinal homeostasis and cancer. Cancer Res 75: 1181-1186. https://doi. org/10.1158/0008-5472.CAN-14-1726

Diefenbacher ME, Popov N, Blake SM, Schülein-Völk C, Nye E, Spencer-Dene B, Jaenicke LA, Eilers M, Behrens A (2014) The deubiquitinase USP28 controls intestinal homeostasis and promotes colorectal cancer. J Clin Invest 124: 3407-3418. https://doi. org/10.1172/JCI73733

Doherty JR, Cleveland JL (2013) Targeting lactate metabolism for cancer therapeutics. J Clin Invest 123: 3685-3692. https://doi. org/10.1172/JCI69741

Elstrom RL, Bauer DE, Buzzai M, Karnauskas R, Harris MH, Plas DR, Zhuang H, Cinalli RM, Alavi A, Rudin CM, Thompson CB (2004) Akt stimulates aerobic glycolysis in cancer cells. Cancer Res 64: 3892-3899. https://doi.org/10.1158/0008-5472.CAN-03-2904

Fleshman JW, Smallwood N (2015) Current concepts in rectal cancer. Clin Colon Rectal Surgery 28: 5-11. https://doi. org/10.1055/s-0035-1545064

He TL, Zhang YJ, Jiang H, Li XH, Zhu H, Zheng KL (2015) The c-Myc-LDHA axis positively regulates aerobic glycolysis and promotes tumor progression in pancreatic cancer. Med Oncol Northwood, London, England). 32: 187-187. https://doi.org/10.1007/s12032-0150633-8

Hu T, Liu H, Liang Z, Wang F, Zhou C, Zheng X, Zhang Y, Song Y, Hu J, He X, Xiao J, King RJ, Wu X, Lan P (2020) Tumor-intrinsic CD47 signal regulates glycolysis and promotes colorectal cancer cell growth and metastasis. Theranostics 10: 4056-4072. https://doi. org $/ 10.7150 /$ thno. 40860

Li Q, Wei P, Wu J, Zhang M, Li G, Li Y, Xu Y, Li X, Xie D, Cai S, Xie K, Li D (2019) The FOXC1/FBP1 signaling axis promotes colorectal cancer proliferation by enhancing the Warburg effect. Oncogene 38: 483-496. https://doi.org/10.1038/s41388-018-0469-8

Liu J, Zhang Z, Li X, Chen J, Wang G, Tian Z, Qian M, Chen Z, Guo $H$, Tang G, Huang W, Tian D, Wang D, Nie Y, Fan D, Wu K, Xia L (2018) Forkhead box C1 promotes colorectal cancer metastasis through transactivating ITGA7 and FGFR4 expression. Oncogene 37: 5477-5491. https://doi.org/10.1038/s41388-018-0355-4

Lu J (2019) The Warburg metabolism fuels tumor metastasis. Cancer Metastasis Rev 38: 157-164. https://doi.org/10.1007/s10555-01909794-5

Pan J, Deng Q, Jiang C, Wang X, Niu T, Li H, Chen T, Jin J, Pan W, Cai X, Yang X, Lu M, Xiao J, Wang P (2015) USP37 directly deubiquitinates and stabilizes c-Myc in lung cancer. Oncogene 34: 3957-3967. https://doi.org/10.1038/onc.2014.327

Popov N, Herold S, Llamazares M, Schulein C, Eilers M (2007) Fbw7 and Usp28 regulate myc protein stability in response to DNA damage. Cell Cycle 6: 2327-2331. https://doi.org/10.4161/cc.6.19.4804

Prenen H, Vecchione L, Van Cutsem E (2013) Role of targeted agents in metastatic colorectal cancer. Targeted Oncol 8: 83-96. https://doi. org/10.1007/s11523-013-0281-x

Yang SZ, Yu JJ, Mao YR (2019) Pregnancy complicated with colon cancer: a case report and review of the literature. Clin Exp Obstetrics Gynecol 46: 1028-1030. https://doi.org/10.12891/ceog4920.2019

Sacco JJ, Coulson JM, Clague MJ, Urbé S (2010) Emerging roles of deubiquitinases in cancer-associated pathways. IUBMB Life 62: 140157. https://doi.org/10.1002/iub.300

Satoh K, Yachida S, Sugimoto M, Oshima M, Nakagawa T, Akamoto S, Tabata S, Saitoh K, Kato K, Sato S, Igarashi K, Aizawa Y, Kajino-Sakamoto R, Kojima Y, Fujishita T, Enomoto A, Hirayama A, Ishikawa T, Taketo MM, Kushida Y, Haba R, Okano K, Tomita M, Suzuki Y, Fukuda S, Aoki M, Soga T (2017) Global metabolic reprogramming of colorectal cancer occurs at adenoma stage and is induced by MYC. Proc Natl Acad Sci U S A 114: E7697-E7706. https://doi.org/10.1073/pnas.1710366114

Sheng Y, Saridakis V, Sarkari F, Duan S, Wu T, Arrowsmith CH, Frappier L (2006) Molecular recognition of $\mathrm{p} 53$ and MDM2 by USP7/ HAUSP. Nat Struct Mol Biol 13: 285-291. https://doi.org/10.1038/ nsmb1067

Soga T (2013) Cancer metabolism: key players in metabolic reprogramming. Cancer Sci 104: 275-281. https://doi.org/10.1111/cas.12085 
Wang H, Meng Q, Ding Y, Xiong M, Zhu M, Yang Y, Su H, Gu L, Xu Y, Shi L, Zhou H, Zhang N. (2020) USP28 and USP25 are downregulated by Vismodegib in vitro and in colorectal cancer cell lines. FEBS J 288: 1325-1342. https://doi.org/10.1111/febs.15461

Wang X, Liu Z, Zhang L, Yang Z, Chen X, Luo J, Zhou Z, Mei X, Yu X, Shao Z, Feng Y, Fu S, Zhang Z, Wei D, Jia L, Ma J, Guo $\mathrm{X}$ (2018) Targeting deubiquitinase USP28 for cancer therapy. Cell Death Dis 9: 186-186. https://doi.org/10.1038/s41419-017-0208-z

Weili Z, Zhikun L, Jianmin W, Qingbao T (2019) Knockdown of USP28 enhances the radiosensitivity of esophageal cancer cells via the c-Myc/hypoxia-inducible factor-1 alpha pathway. I Cell Biochem 120: 201-212. https://doi.org/10.1002/jcb.27305

Wu Y, Wang Y, Yang XH, Kang T, Zhao Y, Wang C, Evers BM, Zhou BP (2013) The deubiquitinase USP28 stabilizes LSD1 and confers stem-cell-like traits to breast cancer cells. Cell Rep 5: 224 236. https://doi.org/10.1016/j.celrep.2013.08.030
Xu X, Li J, Sun X, Guo Y, Chu D, Wei L, Li X, Yang G, Liu X, Yao L, Zhang J, Shen L (2015) Tumor suppressor NDRG2 inhibits glycolysis and glutaminolysis in colorectal cancer cells by repressing c-Myc expression. Oncotarget 6: 26161-26176. https://doi. org/10.18632/oncotarget.4544

Yang WL, Jin G, Li CF, Jeong YS, Moten A, Xu D, Feng Z, Chen W, Cai Z, Darnay B, Gu W, Lin HK (2013) Cycles of ubiquitination and deubiquitination critically regulate growth factor-mediated activation of Akt signaling. Sci Signal 6: ra3-ra3. https://doi. org/10.1126/scisignal.2003197

Zhang J, Zhang P, Wei Y, Piao HL, Wang W, Maddika S, Wang M, Chen D, Sun Y, Hung MC, Chen J, Ma L (2013) Deubiquitylation and stabilization of PTEN by USP13. Nat Cell Biol 15: 1486-1494. https://doi.org/10.1038/ncb2874

Zhang Y, Liao Y, Chen C, Sun W, Sun X, Liu Y, Xu E, Lai M, Zhang $\mathrm{H}$ (2020) p38-regulated FOXC1 stability is required for colorectal cancer metastasis. J Pathol 250: 217-230. https://doi.org/10.1002/ path. 5362 